\title{
Análisis de patrones meteorológicos para temperaturas mínimas en el cantón Riobamba, Ecuador
}

(c) (1) (3) (2)

Meteorological patterns analysis for minimum temperatures for Riobamba city, Ecuador

\author{
Natalia Alexandra Pérez Londo. ${ }^{1}$, Jessica Alexandra Marcatoma Tixi. ${ }^{2}$, Cristina \\ Estefanía Ramos Araujo. ${ }^{3} \&$ Hitler Abdón Guerrero Hidalgo. ${ }^{4}$
}

\begin{abstract}
.
DOI: https://doi.org/10.33262/concienciadigital.v4i3.1.1857

Introduction. Climate change is a subject of special attention, because it can affect the relations between society and nature. Although, when speaking of the Sierra region, a gradual increase in temperatures cannot be guaranteed, by carrying out a localized study it is possible to better understand the reality. Objective. Determining the existence of considerable patterns in the minimum temperature records from 1976 - 2020 for Riobamba city. Methodology. Principal components analysis was performed in order to identify similarities between variables (years) and individuals (months) from minimum temperature. RStudio free software was used through the Factoshiny library. Results. This statistical technique has revealed 3 clusters, group 1: August and September, group
\end{abstract}

\footnotetext{
${ }^{1}$ Escuela Superior Politécnica de Chimborazo, Facultad de Ciencias, Chimborazo, Riobamba, Ecuador, nperez@espoch.edu.ec, https://orcid.org/0000-0001-9068-8790

2 Universidad Nacional de Chimborazo, Facultad de Ingeniería, Chimborazo, Riobamba, Ecuador, jessica.marcatoma@unach.edu.ec, https://orcid.org/0000-0001-9531-3234

${ }^{3}$ Escuela Superior Politécnica de Chimborazo, Facultad de Ciencias, Chimborazo, Riobamba, Ecuador, cristina.ramos@espoch.edu.ec, https://orcid.org/0000-0002-8644-5814

${ }^{4}$ Ministerio del Ambiente, Agua y Transición Ecológica, Dirección Nacional de Normativa y Control Ambiental, Pichincha, Quito, Ecuador, hitler.guerrero@ambiente.gob.ec, https://orcid.org/0000-00020315-3833
} 
2: January, March, April and May, group 3: October and December, which are related to Andean region climate. July however, showed more differences among other months. In the years 1977, 1983, 1986, 1992, 1995,1998, 2005, 2007 the minimum temperature was relevant within the months of January, March, April and May, which were affected by the continental and oceanic air masses that determine El Niño y La Niña phenomena. Conclusion. Further retrospective studies can provide information on the historical effects of these phenomena on the populations and ecosystems of the region.

Keywords: Principal Components, Square Cosines, Minimum Temperature, Correlation.

\section{Resumen.}

Introducción. La variabilidad climática es un tema a tratarse con atención, porque ésta puede afectar las relaciones entre la sociedad y la naturaleza. Si bien, al hablar de la región Sierra, no se puede asegurar un aumento paulatino de las temperaturas, al hacer un estudio localizado se puede comprender mejor la realidad Objetivo. En esta investigación se ha enfocado en determinar la existencia de patrones considerables en los registros de temperatura mínima desde el año de 1976 hasta el 2020 para la cuidad de Riobamba. Metodología. Se ha utilizado el análisis de componentes principales para identificar la similitud entre variables (años) e individuos (meses) de la temperatura mínima, empleando el software libre RStudio mediante la librería Factoshiny. Resultados. Esta técnica estadística ha evidenciado 3 agrupamientos, grupo 1: agosto y septiembre, grupo 2: enero, marzo, abril y mayo, grupo 3: octubre y diciembre, que están relacionados al clima de la región andina, se ha observado una diferencia en el mes de julio. En los años 1977, 1983, 1986, 1992, 1995,1998, 2005, 2007 la temperatura mínima es relevante dentro de los meses de enero, marzo, abril y mayo, que se ven afectadas por las masas continental y oceánica que determinan el fenómeno del Niño y la Niña. Conclusión. El estudio retrospectivo de estos ciclos puede aportar información sobre los efectos históricos de estos fenómenos sobre las poblaciones y los ecosistemas de la región.

Palabras clave: Componentes principales, cosenos cuadrados, temperatura mínima, correlación.

\section{Introducción.}

En la actualidad, de acuerdo al Grupo Intergubernamental de Expertos sobre el Cambio Climático (IPCC), quien es la fuente científica oficial en temas de cambio climático, presenta a éste como "la variación del estado del clima identificable (por ejemplo, mediante pruebas estadísticas sobre datos recopilados en todo el mundo)" (Intergovernmental Panel on Climate Change [IPCC], 2014); muchas veces, influenciado por las actividades antropogénicas como la utilización de combustibles fósiles, explotación de bosques y muchas más acciones consecuencia de los modelos de crecimiento económico que persigue cada región del planeta (Venegas Zapata, 2020). 
La variabilidad climática es un tema a tratarse con atención, porque ésta puede afectar las relaciones entre la sociedad y la naturaleza (Zavgoroniaya, 2011). Si bien, al hablar de la Bioregión Andes, no se puede asegurar un aumento paulatino de las temperaturas, al hacer un estudio localizado se puede comprender mejor la realidad (Toainga Oñate 2015). Un tipo de variabilidad asociada a los cambios en el clima es el evento denominado "Fenómeno del Niño", que no solo se presenta y repercute en el Ecuador y Sudamérica, sino también en todo el globo terrestre (Hernández 2006).

Para el caso de la ciudad de Riobamba, sus vientos, pueden producir una sensación térmica de casi $0{ }^{\circ} \mathrm{C}$ en algunas épocas del año; la máxima temperatura diaria puede alcanzar entre los $25{ }^{\circ} \mathrm{C}$ a $27{ }^{\circ} \mathrm{C}$, rara vez se han registrado temperaturas por encima de los $27{ }^{\circ} \mathrm{C}$. En septiembre de 2009 se registró una temperatura récord de $29{ }^{\circ} \mathrm{C}$. Debido a que el cantón se caracteriza por ser ganadero y agrícola, es importante analizar el comportamiento meteorológico (Toainga Oñate, 2015).

Las técnicas para llevar a cabo un análisis climático, generalmente se basan en la aplicación de los parámetros de la estadística descriptiva, es decir la media, mediana, desviación estándar, varianza, coeficiente de variación y los valores mínimos y máximos correspondientes a las variables climatológicas seleccionadas para un determinado período de registros (Pineda, et al., 2006).

En este trabajo se definen la variable meteorológica Temperatura mínima; dicha variable ha sido tomada desde el año 1976 hasta el año 2020 y tiene por objeto determinar si existen patrones notables en los registros de temperatura mínima para la ciudad de Riobamba y que puedan ser interpretados como indicadores de cambio climático.

\section{Metodologia.}

El estudio fue cuantitativo y transversal, en función al tipo de investigación, manipulación de variables y línea de tiempo respectivamente (Haro-Rivera et al., 2020) (Patten y Newhart, 2017).

\section{Área de estudio}

El estudio se realizó en la estación meteorológica ESPOCH de la ciudad de Riobamba, provincia de Chimborazo, que se caracteriza por su relieve irregular, típico de la zona central de los Andes centrales ecuatorianos. Se localiza a $211 \mathrm{~km}$ al sur de la ciudad de Quito, a una elevación de $2850 \mathrm{msnm}$, en una latitud 9817285 y longitud 757452, en coordenadas WGS 84 UTM zona 17 Sur (ver Figura 1). En cuanto al ombrotipo y al termotipo, en la zona se identifican como "Seco Superior" y "Mesotropical Inferior" respectivamente (Ministerio del Ambiente Ecuador [MAE], 2012) los registros de 2016 indican una temperatura media anual de $13,6{ }^{\circ} \mathrm{C}$, precipitación anual de 537,2 mm y la humedad relativa de 59\%. Los datos utilizados en la presente investigación fueron obtenidos de la estación meteorológica ESPOCH, código M1036 de la red de instalaciones del Instituto Nacional de Meteorología e Hidrología (INAMHI); la cual cuenta con registros desde su instalación en abril de 1975 y a partir de enero de 1976 
empieza a publicar su boletín meteorológico en forma ininterrumpida hasta la fecha del presente estudio (Pérez et al., 2020).

Figura 1

Estación Meteorológica de Recursos Naturales

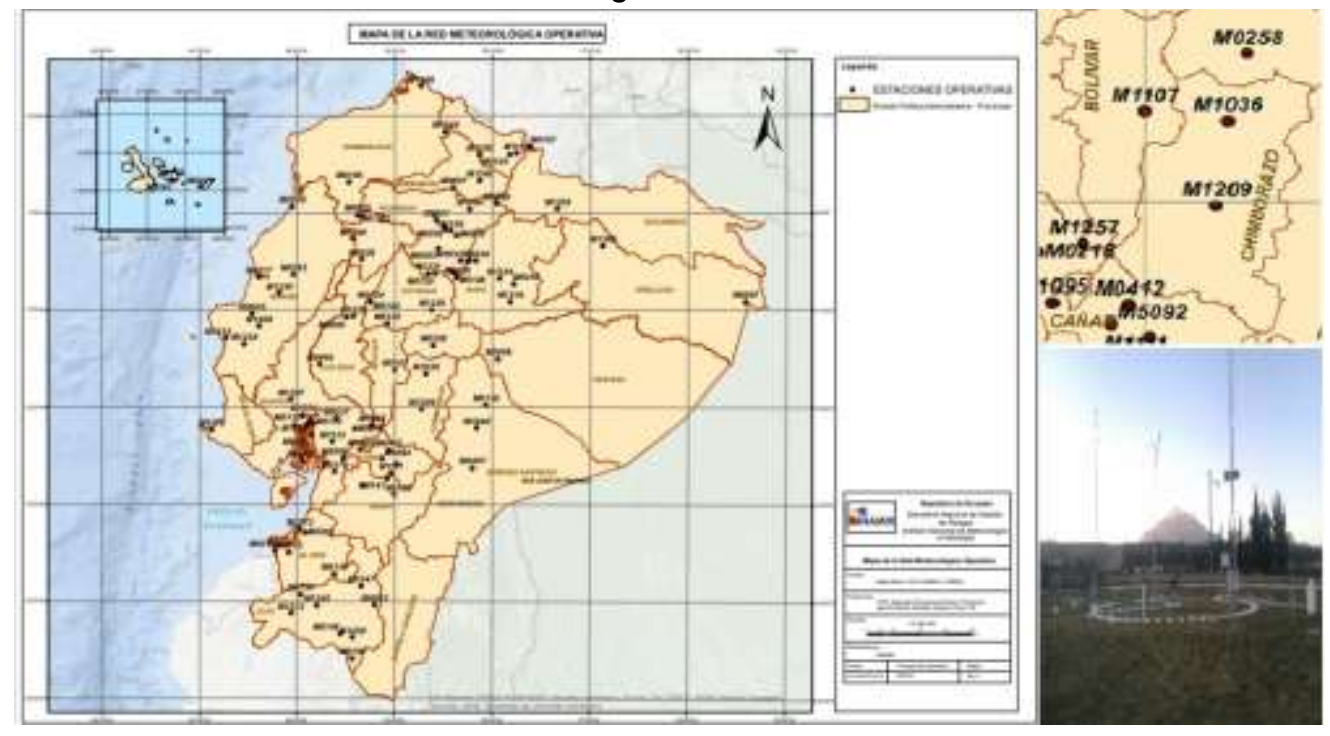

Nota: Ubicación y código de la Estación meteorológica de Recursos Naturales Fuente: Instituto Nacional de Meteorología e Hidrología (INAMHI, 2020)

\section{Instrumentos de recolección de datos}

Esta estación meteorológica cuenta con dos piranómetros SR11-10-Hukseflux, uno de ellos provisto de un anillo de sombra para el registro de radiación difusa, pluviómetro, anemómetro ultrasónico 8500 de marca Vaisalia, barómetro QML 201C de marca Vaisalia, termómetro HMP155 de marca Vaisalia, además de sensores de perfil de temperatura de suelo. Los registros se realizaron de forma manual por técnicos expertos constituyéndose en una de las estaciones meteorológicas con fuente de información histórica (45 años) en la Provincia de Chimborazo (Pérez et al., 2020).

\section{Análisis Estadístico y relleno de datos faltantes}

Los datos de temperatura mínima se registran en fichas técnicas los cuales son tabulados manualmente en grados Celsius $\left({ }^{\circ} \mathrm{C}\right)$ desde 1976 hasta 2020, se realizó el análisis exploratorio (Detección de datos anómalos univariante), además se efectuó un relleno de datos mediante un técnica estadística, para lo cual, la serie debía tener como máximo de 6 meses ausentes por año (Gutiérrez, 2003). Los años que no cumplen con el requisito fueron separados de la base de datos, para mostrar los resultados y propiciar una idea clara del comportamiento de la temperatura mínima a través del tiempo en la Estación meteorológica estudiada.

\section{Análisis de componentes principales (ACP)}

El ACP permitirá la estructuración del conjunto de datos de la estación de estudio durante los 42 años, cuya distribución de probabilidades no necesita ser conocida. En este análisis 
cada estación contribuye con un peso dado (autovalor) en cada uno de los patrones geográficos dominantes. De esta forma puede establecerse la contribución de cada registro individual en el patrón correspondiente (Gutiérrez, 2003).

Las componentes se determinan mediante combinación lineal de las variables originales mismas que deben ser linealmente independientes (Satrustegui, et al., 2014).

Consideremos una tabla de datos $\mathrm{X}$ con $\mathrm{n}$ individuos, se busca un subespacio qdimensional; generalmente un plano, tal que la proyección ortogonal de los n puntos sobre el sub espacio tienen varianza máxima. El objetivo es conservar la información más importante reduciendo la tabla de datos en un conjunto pequeño de nuevas variables, mismo que toma el nombre de componentes principales (Haro-Rivera et al. 2020).

La primera componente principal se define como la combinación lineal de las variables originales que tiene varianza máxima. Los valores en este primer componente de los $n$ individuos se representarán por (Peña, 2002):

$$
z_{1}=X a_{1}
$$

Como las variables originales tienen media cero también $z_{1}$ tendrá media nula. Su varianza será (Peña, 2002):

$$
\frac{1}{n} z_{1}^{\prime} z_{1}=\frac{1}{n} a_{1}^{\prime} X^{\prime} X a_{1}=a_{1}^{\prime} S a_{1}
$$

Donde $S$ es la matriz de varianza y covarianzas de las observaciones. Es obvio que podemos maximizar la varianza sin límite aumentando el módulo del vector $a_{1}$. Para que la maximización de la ecuación 2 tenga solución debemos imponer una restricción al módulo del vector $a_{1}, \mathrm{y}$, sin pérdida de generalidad, impondremos que $a_{1}^{\prime} a_{1}=1$. Introduciremos esta restricción mediante el multiplicador de Lagrange (Peña, 2002):

$$
M=a_{1}^{\prime} S a_{1}-\lambda\left(a_{1}^{\prime} a_{1}-1\right)
$$

Y maximizaremos esta de la forma habitual derivando respecto a los componentes de $a_{1} \mathrm{e}$ igualando a cero. Entonces (Peña, 2002):

$$
\frac{\partial M}{\partial a_{1}}=2 S a_{1}-2 \lambda a_{1}=0
$$

Cuya solución es:

$$
S a_{1}=\lambda a_{1}
$$

Que implica que $a_{1}$ es un vector propio de la matriz $S$, y $\lambda$ su correspondiente valor propio. Para determinar qué valor propio de $S$ es la solución de la ecuación 5, multiplicando por la izquierda por $a_{1}^{\prime}$ esta ecuación (Peña 2002):

$$
a_{1}^{\prime} S a_{1}=\lambda a_{1}^{\prime} a_{1}=\lambda
$$


Y concluimos, por ecuación 2, que $\lambda$ es la varianza de $z_{1}$. Como ésta es la cantidad que queremos maximizar, $\lambda$ será el mayor valor propio de la matriz $S$. Su vector asociado, $a_{1}$, define los coeficientes de cada variable en la primera componente principal (Peña 2002).

Vamos a obtener el mejor plano de proyección de las variables $X$. Lo calcularemos estableciendo como función objetivo que la suma de las varianzas de $z_{1}=X a_{1}$ y $z_{2}=$ $X a_{2}$ sea máxima, donde $a_{1}$ y $a_{2}$ son los vectores que definen el plano. La función objetivo será (Peña 2002):

$$
\emptyset=a_{1}^{\prime} S a_{1}+a_{2}^{\prime} S a_{2}-\lambda_{1}\left(a_{1}^{\prime} a_{1}-1\right)-\lambda_{2}\left(a_{2}^{\prime} a_{2}-1\right)
$$

Que incorpora las restricciones de que las direcciones deben de tener módulo unitario $a_{i}^{\prime} a_{l}=1, i=1,2$. Derivando e igualando a cero:(Peña 2002)

$$
\begin{gathered}
\frac{\partial \emptyset}{\partial a_{1}}=2 S a_{1}-2 \lambda_{1} a_{1}=0 \\
\frac{\partial \emptyset}{\partial a_{2}}=2 S a_{2}-2 \lambda_{2} a_{2}=0
\end{gathered}
$$

La solución de este sistema es:

$$
\begin{aligned}
& S a_{1}=\lambda_{1} a_{1} \\
& S a_{2}=\lambda_{2} a_{2}
\end{aligned}
$$

Que indica que $a_{1} y a_{2}$ deben ser vectores propios de $S$. Tomando los vectores propios de norma una y sustituyendo en la ecuación 7, se obtiene que, en el máximo, la función objetivo es:

$$
\emptyset=\lambda_{1}+\lambda_{2}
$$

Es claro que $\lambda_{1}$ y $\lambda_{2}$ deben ser los dos autovalores mayores de la matriz $S$ y $a_{1}$ y $a_{2}$ sus correspondientes autovectores. Observemos que la covarianza entre $z_{1} y z_{2}$, dada por $a_{1}^{\prime} S a_{2}$ es cero ya que $a_{1}^{\prime} a_{2}=0$, y las variables $z_{1} y z_{2}$ estarán incorreladas (Peña, 2002).

El ACP es una primera técnica que se emplea para determinar variables ocultas que puede generar inconsistencia en el conjunto de datos (Peña, 2002).

El análisis de componentes principales se realizó mediante la librería Factoshiny de R, y para ello se procedió a cargar el fichero y a seleccionar ACP (Haro-Rivera et al., 2020).

Para comprobar que la matriz de correlaciones se ajuste a la matriz identidad (I), es decir ausencia de correlación significativa entre las variables. Esto significa que la nube de puntos se ajustara a una esfera perfecta para lo cual se aplica el test de Esfericidad de Bartlett, expresando así la hipótesis (Urrutia y Reiner Palomino 2010):

$$
H_{0} \text { : el determinante de la matriz de correlaciones es } 1
$$


$H_{1}$ : el determinante de la matriz de correlaciones es diferente de 1

A valores altos, el test rechaza la hipótesis nula, lo que determina que el ACP no es adecuado; pues la matriz de correlaciones no es la matriz identidad (Vaca et al., 2015).

Para determinar si las correlaciones entre las variables son suficientemente pequeñas, se empleó la medida de la adecuación muestral de Kaiser-Meyer-Olkin (KMO), (Urrutia \& Reiner Palomino , 2010). Este estadístico varía entre 0 y 1 , y considera que si $1 \leq$ $K M O \leq 0.9$ el modelo es muy bueno y conforme éste decrece el modelo es considerado como inaceptable (Urrutia y Reiner Palomino 2010) (Haro-Rivera et al., 2020).

El número de componentes se determinó mediante el gráfico de sedimentación, mismo que muestra la cantidad de varianza explicada. El criterio empleado fue calcular el promedio de los autovalores y considerar la matriz de correlaciones, el cual debe ser 1 (KMO) (Ledesma et al., 2007).

La interpretación de las componentes se efectúa mediante la sobre posición de plano principal y el círculo de correlación, lo cual permite interpretar a que grupo pertenecen los individuos (Haro-Rivera et al., 2020) (Girón et al., 2007).

Una manera de determinar las calidades de los individuos y las variables; es calculando los cosenos cuadrados, (Díaz Guevara, 2013). Si $Q € M_{n x m}$, los cosenos están dados por la ecuación:

$$
Q_{i r}=\frac{\left(c_{i, r}\right)^{2}}{\sum_{i=1}^{m}\left(x_{i j}\right)^{2}} \quad \text { para } i=1,2, \ldots, n ; r=1,2, \ldots, m
$$

Sean $a y b$ dos individuos, con $\hat{a} y \hat{b}$ las proyecciones sobre un plano principal, figura 3; se pue de observar que representa mejor a $a$ que lo que lo hace con respecto a b; pues se observa un vector más grande, por lo que podemos decir que a y b están mejor representados si $\alpha$ y $\beta$ son pequeños. Sabemos que si un ángulo tiende a cero entonces su coseno tiende a 1 , por lo que podemos calcular:

$$
\begin{gathered}
\cos (a)=\frac{\|\hat{a}\|}{\|a\|} \\
\cos ^{2}(a)=\frac{\|\hat{a}\|^{2}}{\|a\|^{2}}
\end{gathered}
$$

\section{Resultados.}

Pérez, Mullo y Marcatoma (2020), han determinado una tendencia creciente de la Temperatura mínima anual en la ciudad de Riobamba (Pérez et al. 2020), en base a ese resultado, se ha considerado el Análisis de Componente Principales para esta variable. La prueba de Barlett de la figura 2, se puede observar un p-valor menor al nivel de significancia 0.05 , por consecuente, se rechaza la hipótesis nula de esfericidad y se concluye que el análisis de componentes principales se puede aplicar. 


\section{Figura 2}

Test de Barlett

Bartlett test of homogeneity of variances

data: datos[, 2:42]

Bartlett's K-squared $=60.287, \mathrm{df}=40, \mathrm{p}$-value $=0.02062$

Nota: El test de Barlett obtenido del Software libre RStudio

Fuente: Elaboración propia

\section{Figura 3}

Gráfico de sedimentación de temperatura mínima

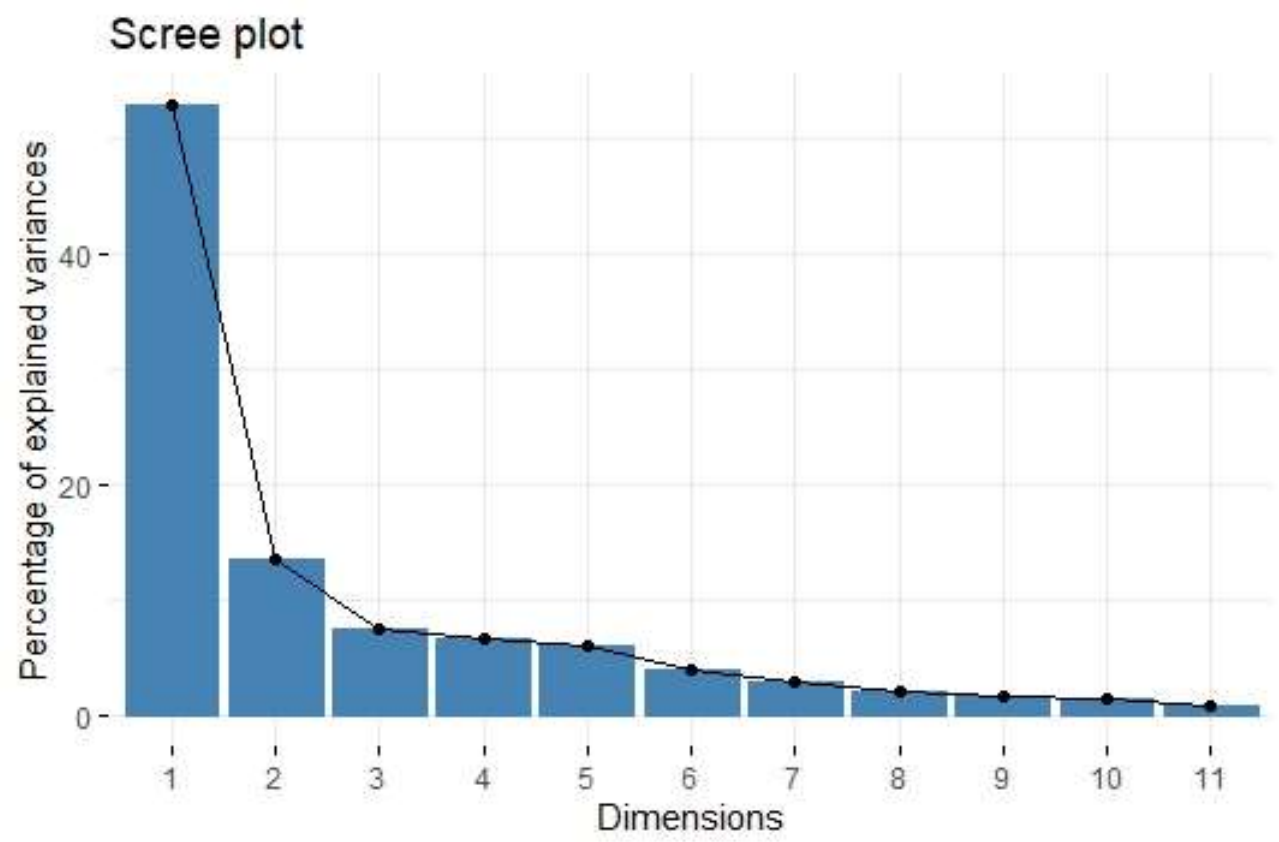

Nota: Porcentaje de la varianza explicada según el número de componentes

Fuente: Elaboración propia

En la figura 3, el gráfico de sedimentación ha permitido identificar dos componentes principales obteniendo, un $66.68 \%$ de la información del conjunto de datos; de la misma forma se puede apreciar en la tabla 1.

Tabla 1

Varianza explicada de la variable temperatura mínima

\begin{tabular}{cccc}
\hline Componentes & Autovalores & $\begin{array}{c}\text { Porcentaje de la } \\
\text { varianza }\end{array}$ & $\begin{array}{c}\text { Porcentaje de la } \\
\text { varianza acumulada }\end{array}$ \\
\hline comp 1 & 21,7539834 & 53,0584961 & 53,0585 \\
comp 2 & 5,5850852 & 13,6221591 & 66,68066 \\
comp 3 & 3,0959147 & 7,5510113 & 74,23167 \\
comp 4 & 2,7302882 & 6,6592394 & 80,89091 \\
comp 5 & 2,5000811 & 6,0977589 & 86,98866 \\
\hline
\end{tabular}


Tabla 1

Varianza explicada de la variable temperatura mínima (continuación)

\begin{tabular}{cccc}
\hline Componentes & Autovalores & $\begin{array}{c}\text { Porcentaje de la } \\
\text { varianza }\end{array}$ & $\begin{array}{c}\text { Porcentaje de la } \\
\text { varianza acumulada }\end{array}$ \\
\hline comp 6 & 1,6517315 & 4,0286133 & 91,01728 \\
comp 7 & 1,2098559 & 2,9508681 & 93,96815 \\
comp 8 & 0,8351241 & 2,036888 & 96,00503 \\
comp 9 & 0,7014022 & 1,710737 & 97,71577 \\
comp 10 & 0,6016442 & 1,4674249 & 99,1832 \\
comp 11 & 0,3348896 & 0,8168038 & 100 \\
\hline
\end{tabular}

Fuente: Elaboración propia

Se ha realizado los siguientes gráficos para la variable temperatura mínima:

Figura 4.

Gráfico plano principal de temperatura mínima

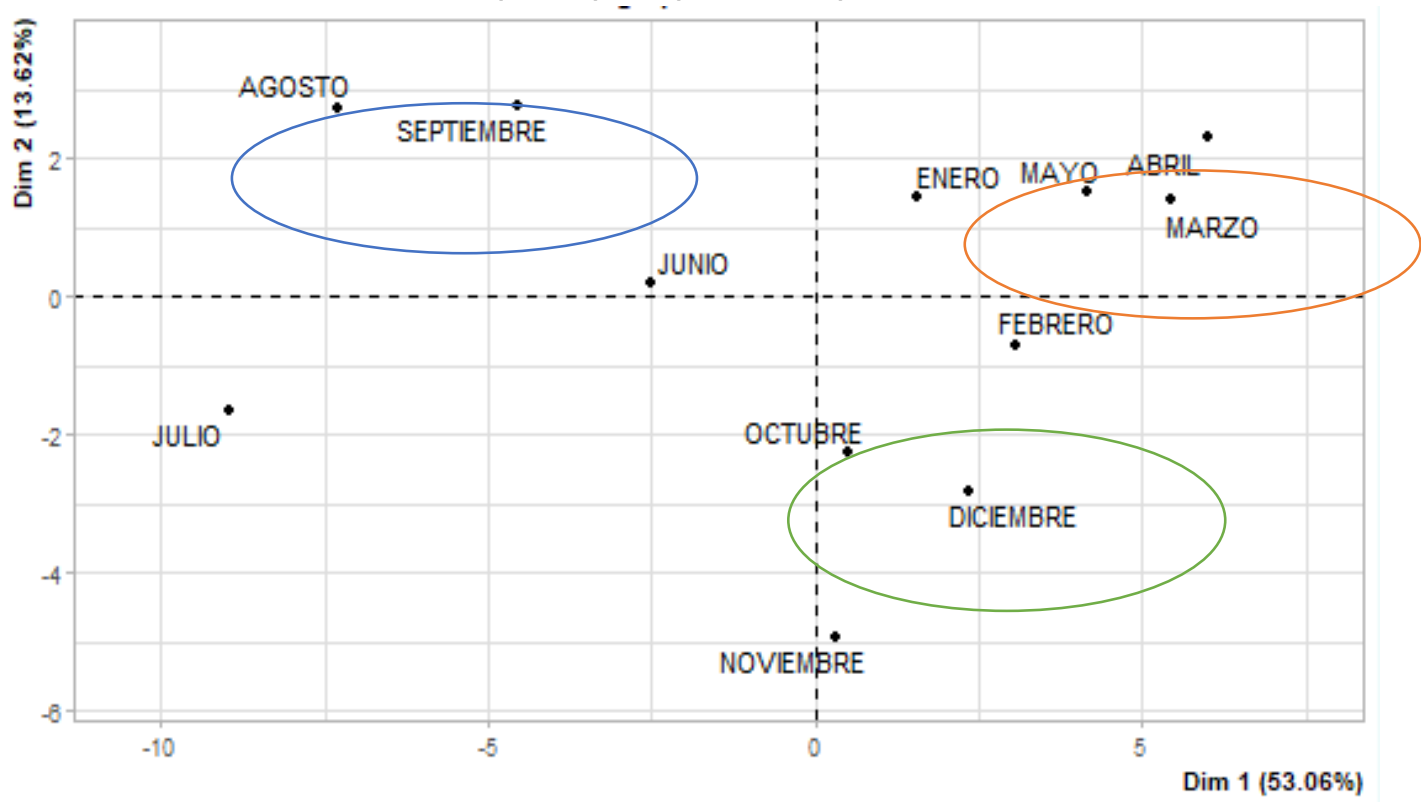

Nota: Gráfico de plano principal de temperatura mínima donde los individuos está representado por los meses

Fuente: Elaboración propia

En el plano principal descrito en la figura 4, se puede apreciar que la temperatura mínima es similar en tres grupos, los que se describe a continuación: grupo 1 (azul); agosto y septiembre, grupo 2 (rojo); enero, marzo, abril y mayo, grupo 3 (verde); octubre y diciembre. Además, se observa que febrero, junio, julio y noviembre la temperatura mínima tiene un comportamiento diferente a los grupos formados. 
Figura 5

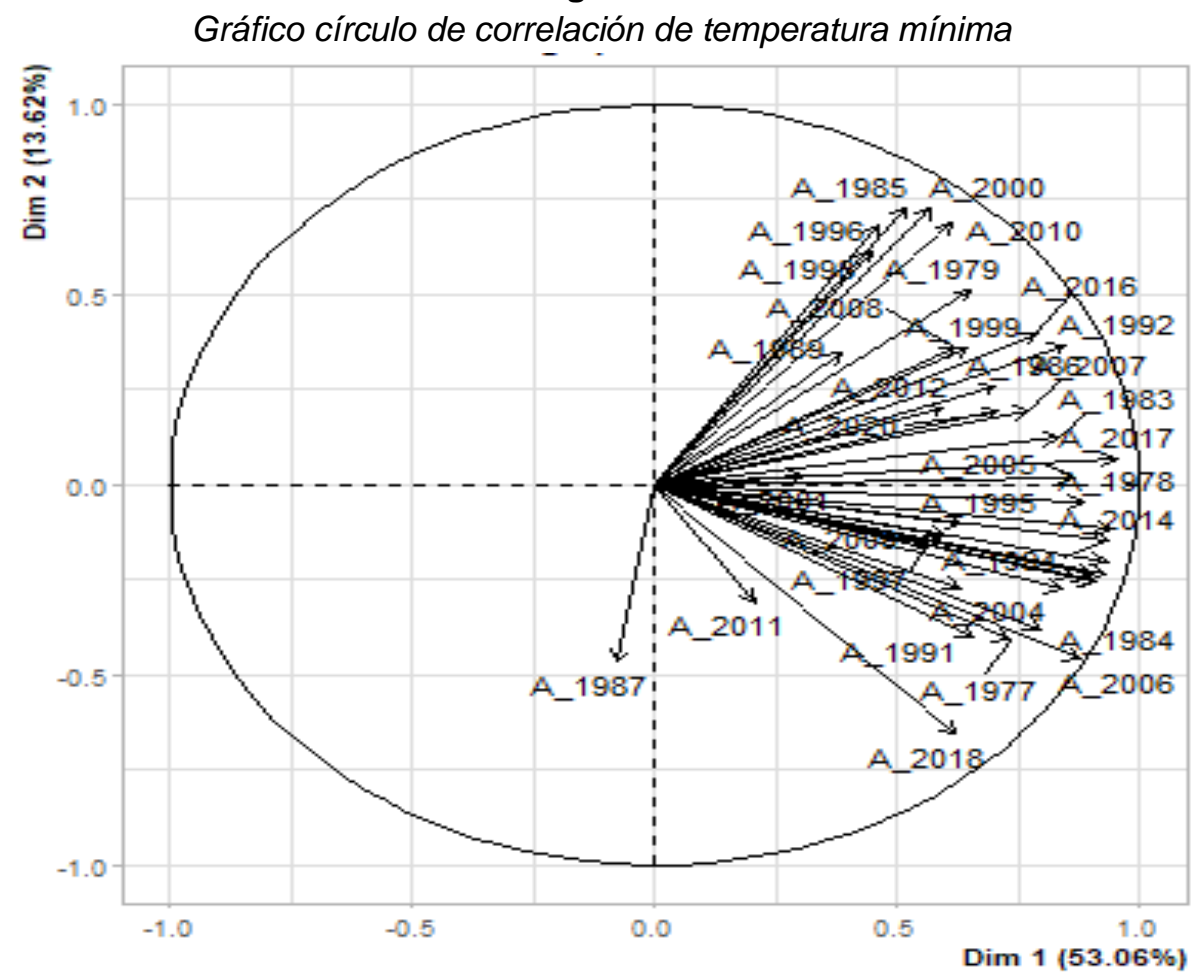

Nota: Círculo de correlación de temperatura mínima según los años

Fuente: Elaboración propia

En el círculo de correlación indicado en la figura 5, se puede considerar que una correlación alta y positiva de temperatura mínima en los años 1985 y 1996, así también como en 1986 y 1992. No existe una correlación de la temperatura mínima entre 1985 y 2018 al igual que entre 1989 y 2011.

En concordancia a la posición del círculo y el plano principal se puede apreciar que la temperatura mínima en los meses de enero, marzo, abril y mayo es superior en los años 1985,1996, 2000, 2007, 2008, 2010, 2016, entre otros. Se determina que existe una relación negativa con el mes de julio, en donde se ha registrado las temperaturas mínimas más bajas de cada año, con una media de $6.76{ }^{\circ} \mathrm{C}$ para el periodo evaluado, se puede observar en la figura 6.

Tabla 2

Promedio multianual de temperatura mínima

\begin{tabular}{|c|c|}
\hline Meses & $\begin{array}{c}\text { Promedio Mensual } \\
\text { Multianual de } \\
\text { Temperatura Mínima }\end{array}$ \\
\hline Enero & 8,49 \\
\hline Febrero & 8,76 \\
\hline Marzo & 9,13 \\
\hline Abril & 9,20 \\
\hline Мayo & 8,86 \\
\hline Junio & 7,88 \\
\hline
\end{tabular}


Tabla 2

Promedio multianual de temperatura mínima (continuación)

\begin{tabular}{cc}
\hline Meses & $\begin{array}{c}\text { Promedio Mensual } \\
\text { Multianual de } \\
\text { Temperatura Mínima }\end{array}$ \\
\hline Julio & 6,76 \\
Agosto & 6,41 \\
Septiembre & 7,06 \\
Octubre & 8,26 \\
Noviembre & 8,25 \\
Diciembre & 8,59
\end{tabular}

Fuente: Elaboración propia

Figura 6

Gráfica de Barras de Temperatura mínima por meses

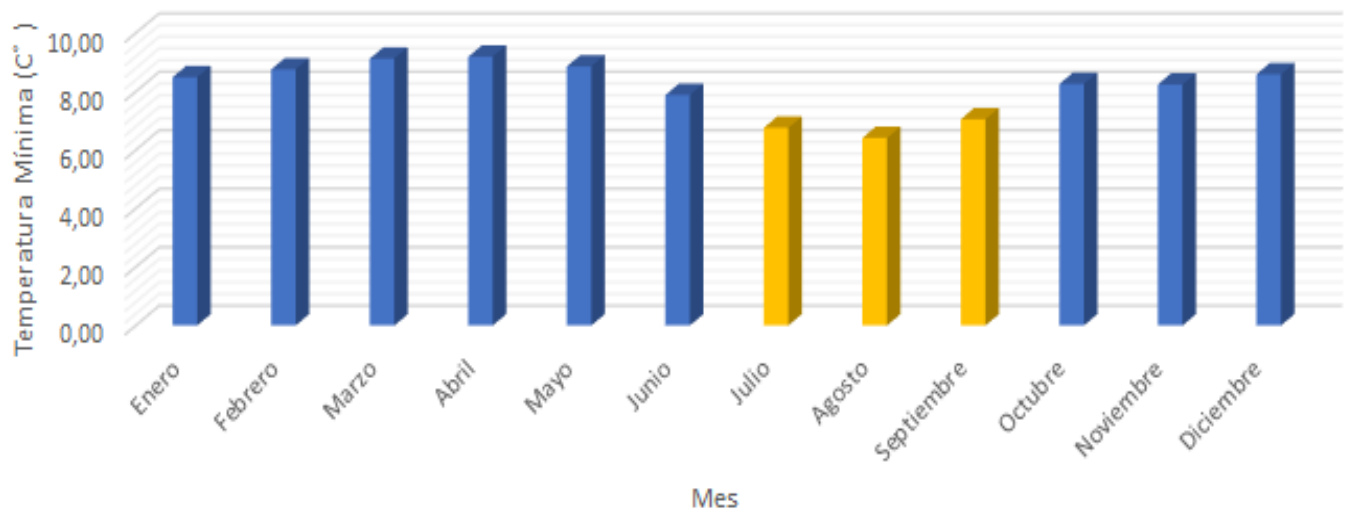

Nota: Comportamiento de la Temperatura mínima de los 42 años de estudio descrito por meses

Fuente: Elaboración propia

La temperatura mínima es significativa en los años 1977, 1983, 1986, 1992, 1995, 1998, 2005 y 2007, dentro de los meses enero, marzo, abril y mayo que coinciden con los días lluviosos (Vuille et al., 2000). Esto se puede influenciar por las masas de aire continental y oceánica que son las responsables del fenómeno del Niño recurrentes cada 4 años, de la misma forma, en los años 1976, 1989, 1999, 2000, 2008 son relevantes en los meses de octubre y diciembre que se ven afectados por el fenómeno de la Niña. (Instituto Oceanográfico de la Armada [INOCAR], 2009). La temperatura mínima en el año de 1987, está separado, se puede decir que en este año se ha presentado datos faltantes, comprobando la falta de robustez de este método (Forsbach, 2005)

A partir de la información disponible de los ecosistemas del Ecuador continental, se ha determinado que el ecosistema que predomina en el área próxima a la estación meteorológica de donde se ha obtenido los datos de temperatura mínima, corresponde al Herbazal de Páramo (Ministerio del Ambiente Ecuador [MAE], 2013) 
Tabla 3

Cosenos cuadrados de los individuos, temperatura mínima

\begin{tabular}{ccc}
\hline Mes & $\begin{array}{c}\text { Primera } \\
\text { componente }\end{array}$ & $\begin{array}{c}\text { Segunda } \\
\text { Componente }\end{array}$ \\
\hline Enero & 0,101 & 0,085 \\
Febrero & 0,481 & 0,024 \\
Marzo & 0,707 & 0,047 \\
Abril & 0,759 & 0,111 \\
Mayo & 0,595 & 0,079 \\
Junio & 0,299 & 0,002 \\
Julio & 0,843 & 0,028 \\
Agosto & 0,733 & 0,102 \\
Septiembre & 0,448 & 0,164 \\
Octubre & 0,010 & 0,226 \\
Noviembre & 0,003 & 0,671 \\
Diciembre & 0,154 & 0,218 \\
\hline
\end{tabular}

Fuente: Elaboración propia

Figura 7

Cosenos cuadrados de los individuos, temperatura mínima

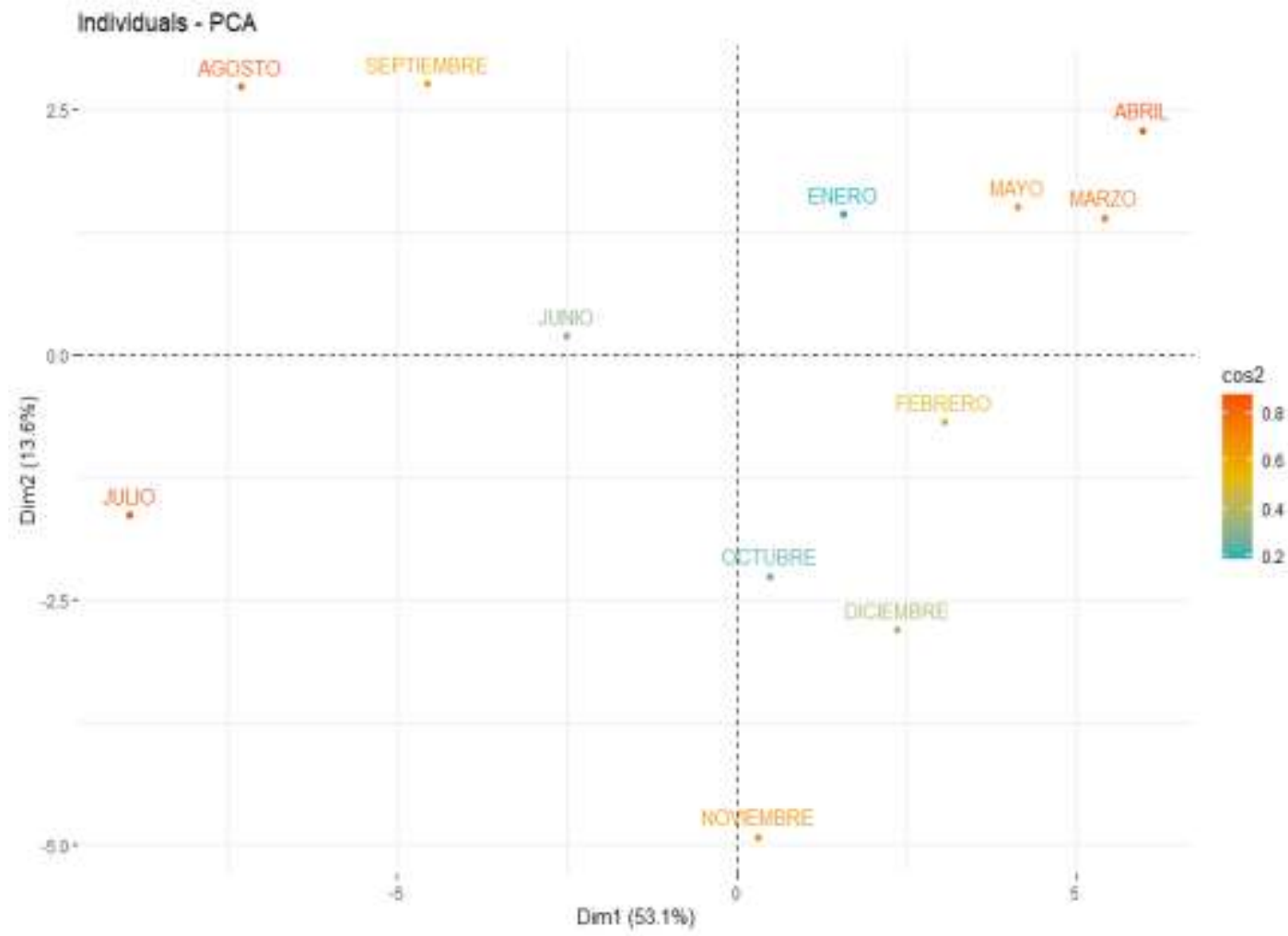

Nota: Representación de los cosenos cuadrados de la Temperatura mínima según los individuos

Fuente: Elaboración propia 
En la tabla 3, los cosenos cuadrados mostraron que el individuo con baja representación en la componente 1 es noviembre, mientras que el individuo que mejor representa en la componente 1 es Julio con valores de 0,003 y 0,843 respectivamente, como se puede apreciar en la Figura 7. Para la componente 2, el individuo que mejor representada un coseno cuadrado igual a 0,67 es Noviembre; mientras que junio tienen una representación baja de 0.002 , de la misma forma se puede apreciar en la tabla 3 .

\section{Conclusiones.}

- El método de PCA no es robusto ante datos faltantes, como se puede corroborar en la gráfica círculo de correlación de temperaturas mínimas donde el año de 1987 tiene características diferentes al resto de años, esto es ocasionado por la falta de información.

- En el rango de análisis de 42 años, el mes de julio presenta una particularidad en cuanto a la temperatura mínima, dado que, al aplicar el PCA no se muestra una relación con el resto de meses agrupados. Esto puede explicarse por la variación estacional de temperatura durante el invierno del hemisferio sur, este comportamiento es más evidente al analizar las temperaturas mínimas, porque la temperatura media se ve influenciada por una serie de factores como la orografía típica de los Andes centrales del Ecuador, la cobertura vegetal y la existencia de diferentes pisos climáticos.

- El análisis de componentes principales aplicado a la serie de temperaturas mínimas de la estación ESPOCH para la ciudad de Riobamba, revela un comportamiento de masas de aire que concuerda con el ombrotipo y termotipo determinados para el área, pero que a su vez evidencia las variaciones cíclicas debidas a fenómenos globales del Niño y de la Niña. El estudio retrospectivo de estos ciclos puede aportar información sobre los efectos históricos de estos fenómenos sobre las poblaciones y los ecosistemas de la región.

\section{Referencias bibliográficas.}

Díaz, J. (2013). Cuantificación del perfil del viento hasta $100 \mathrm{~m}$ de altura desde la superficie y su incidencia en la climatología eólica. Terra nueva etapa 29(46):81101. https://www.redalyc.org/revista.oa?id=721\&numero=30181\&tipo=coleccion

Forsbach, L. (2005). Una técnica robusta para Kernel PCA. [Tesis Doctoral, Centro de Investigación en Matemáticas]. https://cimat.repositorioinstitucional.mx/jspui/bitstream/1008/446/1/TE_1501.pd $\mathrm{f}$

Girón, A., Correa, H., y Caicedo Bravo, E. (2007). Procedimiento automático de reducción de variables basado en ACP para el reconocimiento de formas con ultrasonido. Tecnura 10(20):19-30. https://doi.org/10.14483/22487638.6215 
Gutiérrez, L. (2003). Recopilación y análisis de la variación de las temperaturas (período 1965-2001) y las precipitaciones (período 1931-2001) a partir de la información de estaciones meteorológicas de Chile entre los 33.

Haro-Rivera, S., Zúñiga-Lema, L., Meneses-Freire, A., y Escudero-Villa, A. (2020). Determinación del comportamiento meteorológico del viento en la provincia de Chimborazo, ecuador. Perfiles 1(23):26-32.

Hernández, F. (2006). Análisis de la variabilidad climática de la costa ecuatoriana. Acta Oceanográfica del pacifico 13(1):1-6.

Instituto Oceanográfico de la Armada. (2009). El niño y la niña.

I.P.C.C. (2014). Cambio Climático 2014: Informe de Síntesis. Obtenido de.

Pérez, N., Mullo H. y Marcatoma, A. (2020). Análisis del cambio climático en un ecosistema alto. Perfiles 1(23):4-11.

Ledesma, R., Molina, J., y Mora, P. (2007). Desarrollo de técnicas de visualización múltiple en el programa ViSta: ejemplo de aplicación al análisis de componentes principales. Psicothema 19(3):497-505.

M.A.E. (2012). Cartográfica de los Ecosistemas del Ecuador Continental.

M.A.E. (2013). Guía de ecosistemas del ecuador continental.

Peña, D. (2002). Análisis de datos multivariantes. McGraw-Hill.

Pérez, N., Mullo, S., y Marcatoma, A. (2020). Resumen Abstract 4.

Pineda, N., Jaimes, E. y Mendoza, J. (2006). Aplicación del índice de homogeneidad múltiple a datos climatológicos de Venezuela. Interciencia 31(11):817-21.

Satrustegui, K., Gutiérrez-Goiria, J. y Beatriz Goitisolo, L. (2014). Evidencias e interrogantes sobre desarrollo, financiación externa y AOD: un análisis de componentes principales. Revista de economía mundial 36:153-78.

Toainga Oñate, S. (2015). Dinámica de uso del suelo rural en el periodo 1989 - 2009 y la variabilidad climática en comunidades Altoandinas de la provincia de Chimborazo. http://repositorio.puce.edu.ec/handle/22000/12012

Urrutia, J., y Reiner Palomino, L. (2010). Componentes principales en la determinación de estaciones con Patrones homogéneos de temperatura en el Chocó. Scientia et technica 2(45). https://doi.org/10.22517/23447214.315

Vaca, C., Manosalvas Vaca, C. y Quintero, J. (2015). El clima organizacional y la satisfacción laboral: un análisis cuantitativo riguroso de su relación. AD-minister 26:5-15. https://doi.org/10.17230/ad-minister.26.1. 
Venegas Zapata, J. (2020). Análisis estadístico de datos meteorológicos mensuales y diarios en el periodo 2006-2018 para la determinación de variabilidad climática y cambio climático en el Distrito Metropolitano de Quito.

Vuille, M., Bradley, R. y Keimig F. (2000). Climate Variability in the Andes of Ecuador and Its Relation to Tropical Pacific and Atlantic Sea Surface Temperature Anomalies. Journal of Climate 13(14):2520-35. https://doi.org/10.1175/15200442(2000)013<2520:CVITAO>2.0.CO;2

Zavgorodniaya, S. (2011). Análisis aleatorio de la variabilidad climática en el Ecuador en los últimos 30 años. GEOPUCE Revista de la Escuela de Ciencias Geográficas 59-72.

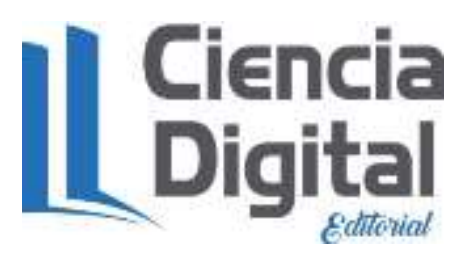




\section{PARA CITAR EL ARTÍCULO INDEXADO.}

Pérez Londo, N. A., Marcatoma Tixi, J. A., Ramos Araujo, C. E., \& Guerrero Hidalgo, H. A. (2021). Análisis de patrones meteorológicos para temperaturas mínimas en el cantón Riobamba, Ecuador . ConcienciaDigital, 4(3.1), 424-439. https://doi.org/10.33262/concienciadigital.v4i3.1.1857

\section{Ciencia \\ Digital \\ Editorial}

El artículo que se publica es de exclusiva responsabilidad de los autores y no necesariamente reflejan el pensamiento de la Revista Conciencia Digital.

El artículo queda en propiedad de la revista y, por tanto, su publicación parcial y/o total en otro medio tiene que ser autorizado por el director de la Revista Conciencia Digital.

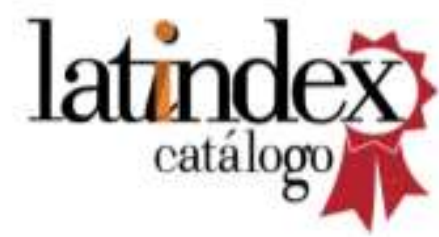

